\section{EL SIGLO XIX EN LOS EXÁMENES DE HISTORIA DE ESPAÑA DE BACHILLERATO. ESTUDIO DE ENUNCIADOS Y CONTENIDOS'}

\author{
Elisa Navarro Medina \\ Departamento de Didáctica y Organización Escolar \\ Universidad de Murcia \\ elisanavarro@um.es \\ José Ramón Urquijo-Goitia \\ Instituto de Historia (CSIC) \\ joseramon.urquïo@cchs.csic.es
}

\author{
Nicolás Martínez Valcárcel \\ Departamento de Didáctica y Organización Escolar \\ Universidad de Murcia
} nicolas@um.es

\begin{abstract}
It is widespread the idea that there is scarce information on the teaching activities in the classroom. As extension, and applied to our field of knowledge, we have few information about what happens in the classrooms in which History is taught and learned. The study of this reality and of the evaluative processes in the classroom gains a special importance for the understanding of the teachinglearning processes. The article that we present gathers a study of the questions made by the teachers in the classrooms to evaluate the contents of the 19th century in the Spanish History courses of the second year of High School in the academic years of 2002-2003 and 2003-2004 in the Autonomous Community of the Murcia Region.
\end{abstract}

KEY WORDS: Evaluation; evaluation in History; typology of the questions; typology of the content.

\section{INTRODUCCIÓN}

Numerosos autores señalan la importancia de la evaluación en los procesos de enseñanza-aprendizaje y de cómo prácticamente influyen, cuando no modelan, toda la estructura educativa. Hay asimismo autores que critican la preeminencia de ésta en los procesos de diseño, pero dichas críticas reconocen en el fondo su papel preponderante en los procesos de enseñanza-aprendizaje. Piaget afirmaba que "el examen escolar constituye un fin en sí mismo, porque domina las preocupaciones del maestro en lugar de favorecer su vocación natural de estimular conciencias
THE NINETEENTH CENTURY IN THE HIGH SCHOOL EXAMINATIONS OF SPANISH HISTORY. A STUDY OVER THE STATEMENTS AND THE CONTENTS

RESUMEN: Es generalizada la idea de que hay escasa información sobre la actividad docente desarrollada en el aula. Como extensión, y aplicado a nuestro campo de conocimiento, se sabe poco de lo que ocurre en las aulas en las que se enseña y aprende Historia. El estudio de esta realidad, y del proceso evaluativo sucedido en ella, cobra una relevancia especial para comprender los procesos de enseñanza-aprendizaje. El artículo que a continuación presentamos recoge un estudio de las preguntas realizadas por los docentes en sus aulas para evaluar los contenidos del siglo XIX en la materia de Historia de España de 2. ${ }^{\circ}$ de Bachillerato en dos cursos académicos, 2002-2003 y 2003-2004, en la Comunidad Autónoma de la Región de la Murcia.

PALABRAS CLAVE: Evaluación; evaluación en Historia; tipología de enunciados; tipología de contenidos.

e inteligencias y orienta todo el trabajo del alumno hacia el resultado artificial del éxito en las pruebas formales, en lugar de centrarse en sus actividades y en su personalidad" (Piaget, 1985, 33).

Cuando pensamos en la evaluación, y en su relación con la dinámica del aula, lo hacemos incluyéndola en el proceso de enseñanza-aprendizaje, no como algo apartado de éste, sino como una fase más dentro de esta práctica. Sin embargo, a pesar de esta influencia de la evaluación en el proceso educativo, destacada por un número importante de autores, en ocasiones podemos encontrar comentarios 
como los de Hargreaves y Ryan en los que se hace referencia a ella "como algo que sigue al aprendizaje, que aparece después de la enseñanza" (Hargreaves y Ryan, 1998, 183); o los de Gimeno y Pérez Gómez (1992) que la consideran una fase más dentro del ciclo de la actividad didáctica y que, además esta fase se encuentra en el último lugar de la enseñanza, con el fin de realizar una revisión del proceso didáctico. Por tanto, la evaluación toma un papel relevante en el aula tanto que, según Santos Guerra (1993), se convierte en la estructura formalizadora de la vida en ella.

Tomando como base estas primeras referencias en torno a la evaluación, el objeto de este trabajo es analizar el tratamiento dado en los exámenes de Historia de $2 .^{\circ}$ de Bachillerato de la CARM a los contenidos del siglo XIX durante dos cursos académicos 2002-2003 y 2003-2004. Para comprender la naturaleza de este estudio es preciso comentar algunas aportaciones metodológicas al respecto.

La dificultad que supone disponer de los exámenes que se realizan en las aulas, puesto que pertenecen a la esfera privada y muchas veces inaccesible, nos llevó a plantearnos la recogida de la información a través de los dos agentes educativos principales: docentes y alumnado. De los primeros de ellos extrajimos los datos mediante el desplazamiento a 3 institutos de la provincia que nos proporcionaron 34 exámenes correspondientes a 8 docentes. Con el permiso de 4 profesores de primer curso de las titulaciones de la Facultad de Educación, de la Universidad de Murcia, explicamos en sus aulas al alumnado la finalidad de nuestra investigación, solicitándoles los exámenes en el caso de que éstos los tuvieran y conservaran, o si podían acceder a ellos por amistad con otros compañeros o con su profesorado del curso de $2 .^{\circ}$ de Bachillerato. Como resultado, logramos que 155 discentes nos aportasen un total de 322 exámenes. La muestra nos ha proporcionado información de 101 de los 127 centros que imparten Bachillerato en la CARM, constituyendo un $79,5 \%$ del total.

Con los documentos logrados por ambas vías alcanzamos un número total de 356 exámenes que contenian el enunciado de una serie de preguntas (1.559 en total) sobre la materia a examinar y donde en ningún caso, se han recogido las respuestas, cuyo acceso está limitado por la ley.

600
Centrándonos en los períodos históricos a los que correspondian estas 1559 cuestiones en los cursos académicos estudiados, 1383 (el 79,35\%) hacian referencia a la España Contemporánea. De ellas, 763 (un 55,17\%) abordan sucesos transcurridos durante el siglo XIX, frente a las 620 cuestiones (el 44,83\%) que lo hacen del siglo XX.

El análisis de la información aportada a través de los exámenes, nos permitió elaborar una base de datos a partir de la cual pudimos categorizar no sólo la tipología de enunciados y su contenido histórico, sino también, las capacidades intelectuales desarrolladas en ellos y el decreto bajo el cual se propusieron. Un ejemplo de examen es el siguiente:

\section{EXAMEN DE HISTORIA Tema $5 \quad 2 .^{\circ}$ Bachillerato}

1. Desarrolla uno de los siguientes temas:

a. La década moderada y su obra legislativa.

b. El bienio progresista y su obra legislativa.

2. Comentario de texto.

Puntos a desarrollar:

a. Realiza un comentario general del siguiente documento.

b. ¿Cuáles fueron los partidos dinásticos que sostuvieron el trono de Isabel II? Describelos.

c. Breve comentario de la figura de Espartero.

\section{Espartero y el partido progresista durante el reinado de Isabel II}

"El partido progresista de 1840, sintiéndose débil, buscó un apoyo, identificó su suerte con la de un soldado: error fatal, casi siempre sin remedio. La fuerza vive de la fuerza, y muere a manos de la fuerza, y cuando ella se ha entronizado, las doctrinas de un partido han cesado de ejercer acción vital, sus sistemas han caducado, en llegando a este punto, no suele haber otro recurso que abrazarse con el ídolo para vivir o morir con él. Espartero era, sin duda, de escasa comprensión política; pero, aún así y todo, era una necesidad para el partido, que le había decretado ovaciones y encumbrándole a la regencia. Los progresistas de la coalición dijeron para sí: 'nosotros somos el pedestal del coloso; retirémonos, y el coloso caerá y se hará pedazos'. Pero no advirtieron que esos pedazos los aplastarían a ellos".

(El pensamiento de la Nación, 8 enero 1845). 
Para detallar nuestro estudio comenzaremos haciendo un repaso de las disposiciones legislativas vigentes en el momento de recogida de información, continuaremos con una descripción de nuestro trabajo y finalizaremos con unas conclusiones que nos permitan establecer la relación existente entre lo legislado y lo que realmente se realiza en el aula.

\section{La Historia en los decretos de currículo NACIONAL Y AUTONÓMICO}

La aprobación de la LOGSE (Ley 1/1990, de 3 de octubre) supuso un cambio notable en el sistema educativo español. En su preámbulo se señalaba como objetivo la concepción de la educación como la herramienta imprescindible para el progreso social de los ciudadanos y la conformación de una identidad personal y comunitaria. La transmisión de los valores más destacados de una sociedad a través de ella y el enriquecimiento académico y personal permite que se conciba como una necesidad inherente al nuevo sistema político y social. En este sentido, la formación de los ciudadanos españoles debe estar encaminada al ejercicio de una democracia responsable, que les proporcione las capacidades propias para la toma de decisiones de forma argumentada y justificada y que, a su vez, les eduque en valores como la tolerancia, el respeto y la solidaridad.

Dentro de esta concepción de la educación como imprescindible, el Bachillerato se articula como el paso entre una enseñanza secundaria y una enseñanza de carácter profesional o universitaria que da al discente la posibilidad de especializarse en un área o temática concreta. Su organización académica, atendiendo a diversas modalidades formativas (Artes, Ciencias de la Naturaleza y de la Salud, Humanidades y Ciencias Sociales), debe poner al alcance del alumnado objetivos y capacidades como el dominio fluido de la lengua española, y la propia de su comunidad autónoma si la hubiera; expresarse con fluidez en una lengua extranjera; ser capaz de analizar y valorar críticamente el mundo contemporáneo; comprender los principios fundamentales de la investigación y el método científico; y consolidar una madurez personal y social que le permita gozar con respeto de su libertad, participando de forma solidaria en el desarrollo de su entorno social. Este período formativo tomará como pilar una educación comprensiva, compatible con una progresiva diversificación elegida de forma personal por cada estudiante, permitiendo acoger sus intereses diferenciados y adaptándose a la pluralidad de sus necesidades y aptitudes.

El desarrollo de dicha ley por el Ministerio de Educación y Ciencia se concretó en el R. D. 1178/1992, de 2 de octubre, por el que se establecen las enseñanzas mínimas del Bachillerato. El estudio de la Historia como disciplina dentro de éste, supone un elemento indispensable para que el alumnado alcance a comprender la importancia de los acontecimientos pasados y su repercusión en la sociedad actual. El ámbito cronológico de la materia queda circunscrito a dos siglos, XIX y XX, decisión que se justifica por la necesidad del discente de realizar un estudio en profundidad de sus raíces más cercanas. Junto a este acotamiento temporal, tampoco ha de olvidarse el enfoque eminentemente político-institucional de la Historia enseñada que permite agrupar los sucesos para facilitar el estudio. En este sentido, el R. D. de 1992 entiende que los objetivos que deben inspirar esta materia se relacionan con la necesidad inherente del alumnado de entender y explicar los hechos relevantes de la España contemporánea, comprendiendo los principales procesos políticos, económicos, sociales y culturales y empleando la terminología básica de ésta que le permita argumentar sus ideas de forma crítica y fundamentada.

Concretando en los contenidos a los que se refiere el $R$. D., se recoge en primer lugar la necesidad de los discentes de conocer y a utilizar las fuentes y procedimientos que permiten acercarse al conocimiento histórico $y$, en segundo lugar, se hace un repaso en profundidad de los hechos sucedidos durante el período contemporáneo, desde el Antiguo Régimen, pasando por las Cortes de Cádiz, el período absolutista de Fernando VII, el régimen liberal de Isabel II y la Restauración y llegando hasta la España del Franquismo y la recuperación democrática. Junto a los contenidos, es preciso mencionar los criterios de evaluación propuestos en dicha norma, remarcando que el proceso evaluativo al que se verá sometido el discente tiene que ver con "reconocer y apreciar, en la Historia de España posterior al siglo XVIII y en cuestiones de actualidad, las huellas de un pasado más lejano" (R. D. 1178/1992, anexo 1, Historia) que le permita conocer este período histórico, sus acontecimientos y procesos políticos, económicos, sociales y culturales más relevantes, a través de su capacidad de 
identificación, análisis y reflexión que le permitan no sólo la adquisición de conceptos sino también la posibilidad de establecer comparaciones y aportaciones de la realidad histórica objeto de estudio.

El traspaso de competencias en materia educativa del Estado a las Comunidades Autónomas se alcanza para la CARM en el año 1999. De esta nueva autonomía educativa se desprende la responsabilidad de cumplir los Reales Decretos establecidos por el MEC, a la vez que se debían ampliar los contenidos para adaptarlos a su realidad social y cultural. Así, en el año 2000, el MEC aprobó el R. D. $3474 / 2000$ en torno a las enseñanzas mínimas del Bachillerato, que modificaba y ampliaba el vigente hasta el momento (R. D. de 1992), y dos años más tarde, en 2002, la CARM aprobó su propia norma (Decreto 113/2002, de 13 de septiembre) por la que ajustaba las ampliaciones de los contenidos mínimos del Bachillerato a su espacio territorial.

El estudio que hemos realizado concierne al R. D. de 1992 y al Decreto de 2002, ya que el análisis Ilevado a cabo por Martínez Molina (2008) puso de manifiesto las escasas modificaciones existentes entre los decretos del 2000 y 2002. Por tanto, una primera y necesaria comparación entre ambos decretos (1992 y 2002) refleja la importante diferencia en la amplitud cronológica de los contenidos a estudiar. Así pues, mientras que el R. D. de 1992 establecía que la enseñanza de la Historia debe centrarse en el aprendizaje de los siglos XIX y XX, el Decreto de la CARM de 2002 contempla que la enseñanza de Historia debe abarcar todos los períodos históricos, desde el proceso de hominización en la Península lbérica hasta la situación político-social actual.

Bajo esta diferencia subyace un cambio de concepción a la hora de abordar los contenidos de Historia. Se pasa de un conocimiento acotado de la misma, en la que el discente sólo debe conocer 2 siglos de nuestro largo pasado, a un conocimiento más amplio y globalizador de éste. Pero esta ampliación de contenidos no conlleva un cambio en profundidad de los aspectos evaluativos planteados por el R. D. de 1992, pues la concepción de éstos desde un carácter global del conocimiento de las raíces de la Historia de España, que permiten comprender los sucesos transcurridos y su repercusión en la actualidad, únicamente quedan modificados en la amplitud conceptual del objeto de estudio.

\section{LOS EXÁMENES COMO INSTRUMENTOS DE evaluación en el aula de Historia de España}

Tomando la legislación como marco de referencia del proceso de enseñanza-aprendizaje del aula, una de las tareas más relevantes de los trabajos de evaluación que los docentes hacen del aprendizaje adquirido por su alumnado, está referida a la obtención de la información, de las evidencias de lo que se ha alcanzado y, lógicamente ésta, será llevada a cabo a través de las técnicas de evaluación. La elección de una técnica u otra estará en función de los

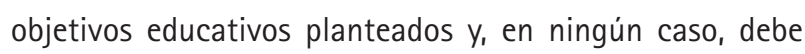
limitarse a una sola, sino que se debe contar con aquellas técnicas que nos permitan obtener la información diversificada necesaria para evaluar el aprendizaje alcanzado por los discentes.

Existen varias definiciones sobre el contenido del término "técnicas de evaluación". Nos inclinamos por la realizada por Martínez Valcárcel donde define el concepto como "instrumentos, situaciones, recursos o procedimientos que se utilizan para obtener información sobre el proceso educativo en general y sobre el proceso de aprendizaje de los alumnos en particular" (Martínez Valcárcel, 2003, 297).

Al igual que la evaluación se entendió en sus comienzos como una medición, los exámenes, como instrumento de obtención de información, encontraron su mayor desarrollo en las sociedades industriales, con el fin de asegurar el ingreso en ciertas profesiones de élite. Desde sus comienzos, éstos colaboraron en el proceso de medición, clasificación y selección no diferenciándose de la evaluación, tal y como actualmente se concibe ésta.

Esta tradición es la que propicia la amplia confianza que los docentes tienen en que la mejor forma de evaluar es el examen, constituyendo esta prueba la base fundamental para la calificación de los estudiantes. Como trabajos significativos que cuestionan esta convicción, podemos señalar las investigaciones de Gullickson (1982) y la de Herman y Dorr-Bremme (1984), las cuales plantearon cuestiones importantes acerca de la calidad de las pruebas efectuadas por el docente y sus implicaciones negativas para la enseñanza y el aprendizaje.

Así pues, cuando los exámenes pasan a constituirse en los instrumentos más importantes de evaluación para el 
alumnado, es necesario plantearse qué tipo de tareas y de procesos cognitivos van a ser los requeridos para superar dichas pruebas (por ejemplo: repetición, crítica, aplicación, creatividad, etc.). Igualmente, cuando el profesor pretende obtener información acerca del rendimiento de sus discentes, ha de preguntarse por qué elige unas técnicas evaluativas y no otras, qué características tienen éstas y qué nivel de coherencia encierran tanto con el método de aprendizaje, como con el de evaluación (por ejemplo, insistencia en la comprensión frente a exámenes de carácter repetitivo) (Santos Guerra, 1993).

Por otra parte, es bien sabido que, para el profesor, cuando hablamos desde la concepción tradicional de lo que es un examen, nos estamos remitiendo habitualmente a exámenes orales, pruebas objetivas o exámenes tipo test y de redacción. Debido a la singularidad de nuestra investigación, y por ser los exámenes de redacción los más utilizados en la asignatura de Historia por parte de los docentes, nos centraremos a continuación en su estudio.

\section{LOS EXÁMENES DE REDACCIÓN Y SU TIPOLOGÍA DE enunciados en la asignatura de Historia}

Los exámenes de redacción son las pruebas más utilizadas a la hora de evaluar al alumnado en la materia de Historia. Este hecho se puede atribuir a la facilidad que supone adaptar el ritmo de evaluación a las posibilidades reales de la enseñanza-aprendizaje (Rodríguez Diéguez, 1980). Más concretamente, estos exámenes consisten en la redacción, articulada en torno a un tema, de un tópico o contenido propuesto al discente, el cual durante cierto tiempo escribe sobre el mismo (Rodríguez Diéguez, 1980). Para muchos profesionales de la enseñanza, este tipo de examen permite la evaluación de unas determinadas capacidades, que otras pruebas como las objetivas u orales, no permiten.

Si bien podemos encontrar diferentes denominaciones y tipologías de estos exámenes (Lafourcade, 1972; Zabalza, 1991; Alonso Tapia, 1997; Rodríguez Neira, 2001; Wragg, 2003; Beltrán Tena y Rodríguez Diéguez, 2004), existe entre algunos de los autores mencionados un cierto consenso a la hora de establecer una designación común. Por ejemplo, Rodríguez Diéguez (1994), Beltrán Tena y Rodríguez
Diéguez (2004), Zabalza (1991) y Alonso Tapia (1997), los denominan exámenes escritos, aunque se pueden observar algunas diferencias en sus clasificaciones internas.

Beltrán Tena y Rodríguez Diéguez (2004) consideran las pruebas de tipo ensayo o redacción como el modelo más convencional y clásico de los exámenes de redacción. Estas pruebas recogen un enunciado que ha de ser desarrollado de modo completo y coherente. Junto a éstas, hacen referencia a la solución de problemas como otra estrategia de evaluación no privativa de las matemáticas o la física. Con ellas, el docente pretende que su alumnado ponga de manifiesto su capacidad de actuación en situaciones conflictivas y las posibilidades de aplicación de las informaciones generalizadoras. Como estrategia más específica, ambos autores definen las cuestiones breves. Su utilización en el aula permite la realización de un recorrido más completo, aunque más conciso, sobre los conocimientos que posee el alumnado facilitando una valoración de la prueba. Para finalizar con la clasificación realizada por los autores mencionados, se recoge la técnica del bosquejo de un tema amplio. En ella la tarea consiste en un esbozo extenso de un tema que por falta de tiempo no puede ser estudiado al completo y que es preciso sintetizar.

Lo más característico de las pruebas libres, como Rodríguez Neira (2001) denomina a estos exámenes, "es la libertad de expresión del alumno que ha de organizar sus propios conocimientos, seleccionar lo más importante, manifestar su creatividad y originalidad, integrando en un todo armónico los elementos de su respuesta". Este autor no establece una clasificación interna clara, sí hace referencia a las preguntas de respuesta abierta, las cuales considera el formato más popular y más utilizado de las pruebas libres, principalmente en la materia de Historia de España en 2. de Bachillerato (Rodríguez Neira, 2001).

Wragg (2003), a diferencia de Rodríguez Neira, denomina a estas pruebas ensayos y realiza una clasificación interna donde señala que las preguntas basadas en ensayos suelen ser o bien de respuesta "limitada" o "cerrada", o bien de respuesta "extendida" o "abierta". Las primeras intentan que el alumnado aborde un tema, o un conjunto de temas, de una manera concreta, sin divagar demasiado (por ej. "Describe dos ejemplos..." o "Analiza los efectos de..."). En cambio, las respuestas abiertas o extendidas ofrecen la oportunidad de revelar una gama de conocimientos más

ARBOR Vol. 187749 mayo-junio [2011] 599-615 ISSN: 0210-1963 
amplia y tienden a plantear preguntas como: "Explica los efectos...".

Por otra parte, Lafourcade (1972) realiza una descripción pormenorizada de las técnicas de evaluación. Más concretamente, y con relación a los exámenes escritos, los denomina pruebas que requieren la elaboración de algún tipo de respuesta, que a su vez forman parte de una clasificación más amplia llamada "pruebas de papel y lápiz".

Por último, tras esta revisión del significado y tipología de los exámenes de redacción, es pertinente hacer referencia a la investigación realizada por Villa y Alonso Tapia (1996). Uno de los propósitos de dicha investigación fue el de analizar el grado en que se apreciaban diferencias en el tipo de estructura formal de examen que los docentes ponen al alumnado para evaluar sus conocimientos. La investigación iba dirigida a las siguientes materias: Ciencias Naturales, Física y Química, Matemáticas, Lengua y Literatura y Ciencias Sociales, de entre las cuales destacan en los exámenes el uso de "preguntas cortas" (se solicita una respuesta bre- ve y concreta) y de "resolución de problemas" (el alumno debe interpretar un enunciado transformándolo en códigos matemáticos para resolver lo formulado en el enunciado). Pero además de estos formatos aparecen otros, aunque de manera menos significativa: las pruebas objetivas, los comentarios de texto, problemas y cómputo. Sin embargo, si nos centramos en la materia que más concierne a nuestro estudio, las Ciencias Sociales, Villa y Alonso señalan que el formato más utilizado en los exámenes de evaluación por parte del profesorado es el de preguntas cortas y, en un número mucho más reducido, las de ensayo, donde el discente tiene que desarrollar un tema sin limitar ni estructurar lo que debe escribir.

Sin lugar a dudas los exámenes, y en el caso de Historia los exámenes de redacción, tienen una gran importancia en el proceso de evaluación y vienen definidos por la tipología de las preguntas que los conforman. Los trabajos anteriormente señalados nos han permitido establecer una categorización de los enunciados de las preguntas recogidas. Con tales datos presentamos la siguiente clasificación:

TABLA 1: RESULTADOS DE LA TIPOLOGÍA DE PREGUNTAS

\begin{tabular}{|c|c|c|c|c|}
\hline Tipología Enunciados & Sub-tipología enunciados & $\mathrm{N} .^{\circ}$ de preguntas & \% Total & $\%$ Relativo \\
\hline \multirow{2}{*}{ Abiertas } & Abiertas no guiadas & 264 & & 90,72 \\
\hline & Abiertas guiadas & 27 & & 9,28 \\
\hline \multicolumn{2}{|l|}{ Total Abiertas } & 291 & 38,14 & 100,00 \\
\hline \multirow{2}{*}{ Acotadas } & Acotadas por signos & 110 & & 44,72 \\
\hline & Acotadas por expresiones & 136 & & 55,28 \\
\hline \multicolumn{2}{|l|}{ Total Acotadas } & 246 & 32,24 & 100,00 \\
\hline \multirow{2}{*}{ Concretas } & Concretas de carácter general & 74 & & 89,16 \\
\hline & Concretas de comparación & 9 & & 10,84 \\
\hline \multicolumn{2}{|l|}{ Total Concretas } & 83 & 10,88 & 100,00 \\
\hline \multirow{2}{*}{ Comentarios de texto } & Comentario no guiado & 81 & & 74,31 \\
\hline & Comentario guiado & 28 & & 25,69 \\
\hline \multicolumn{2}{|l|}{ Total Comentarios de texto } & 109 & 14,29 & 100,00 \\
\hline \multicolumn{2}{|l|}{ Mapas } & 1 & 0,13 & \\
\hline \multicolumn{2}{|l|}{ Vocabulario } & 32 & 4,19 & \\
\hline \multicolumn{2}{|l|}{ Diapositivas } & 1 & 0,13 & \\
\hline \multicolumn{2}{|l|}{ Total } & 763 & 100,00 & \\
\hline
\end{tabular}


Las preguntas abiertas son enunciados de redacción simple y sencilla en los que se pregunta por contenidos amplios y donde no queda definido el modo de abordar su contestación, es decir, pretende que el alumno pueda desarrollar su respuesta libremente, sin límite alguno. La representación de esta tipología en las preguntas estudiadas está en un $38,14 \%$, constituyéndose como la tipología más utilizada en los exámenes de Historia. Dentro de este tipo de preguntas, encontramos dos subcategorías:

Las preguntas no guiadas, con una representación del $90,72 \%$ de la categoría, constituidas simplemente de un enunciado con las características mencionadas:

50.40. El problema agrario.

11.1. Reinado de Fernando VII.

Las preguntas guiadas que, además del enunciado anterior, incluyen un desglose de los puntos a desarrollar y cuentan con un $9,28 \%$ de la categoría:

55.23. La obra reformista de las Cortes de Cádiz. Desarrollar: 1) constitución de 1812: contexto histórico en que es elaborada y promulgada; 2) Resuma brevemente sus contenidos; 3) Enumere las otras leyes básicas aprobadas por esas Cortes y que dieron por resultado el desmantelamiento jurídico del Antiguo Régimen.

5.2. La doble desamortización eclesiástica y civil. Desarrollar: 1) La desamortización de Juan Álvarez Mendizábal: precedentes, desarrollo y balance; 2) La desamortización de Pascual Madoz: desarrollo y balance; 3) ¿Hubo otra desamortización eclesiástica y civil posterior a Mendizábal y Madoz? Razone brevemente la respuesta.

Por su parte, las preguntas acotadas recogen enunciados de redacción simple sobre un tema de contenido amplio que está enmarcado en otro de mayor extensión. Dicha limitación queda reflejada bien por los contenidos a desarrollar o por los signos de puntuación que son introducidos (punto, dos puntos o paréntesis, pero sin utilizar los signos de interrogación). Su representación en nuestro estudio está en un $32,24 \%$, siendo las segundas en importancia. En ellas podemos encontrar dos subcategorias internas:
La acotada por signos formada por un enunciado cuya respuesta libre queda limitada al introducir algunos signos de puntuación como aclaración del contenido general de la pregunta, con un $44,72 \%$ de representación del total de la categoría:

48.18. El comercio: el proteccionismo y el librecambismo.

13.18. Las consecuencias del desastre. La crisis del 98.

Y la acotada por expresiones, siendo aquel enunciado cuya respuesta libre queda limitada al introducir algunas dimensiones de los contenidos que reducen y/o aclaran la respuesta a la pregunta, representada en un $55,28 \%$ de la categoría:

33.10. Consecuencias sociales de la desamortización.

123.2. Medidas económicas del Trienio constitucional.

La tercera tipología en representación es el comentario de texto $(14,29 \%)$. Formado por un texto histórico del cual hay que extraer información sobre un determinado período, acontecimiento o hecho concreto, encontramos en él dos subcategorías:

Los comentarios no guiados, en los cuales únicamente aparece el artículo o texto en cuestión, sin más indicaciones, representados en un $74,31 \%$ :

2.2. El funcionamiento del sistema político de la Restauración: oligarquía y caciquismo. Comentario de texto:

"Los elementos componentes de nuestro régimen oligárquico (...) son tres: 1. ${ }^{\circ}$ Los oligarcas (los Ilamados primates), prohombres o notables de cada bando, que se forman su "plana mayor", residentes ordinariamente en el centro. 2. Los caciques, de primero, segundo ulterior grado, diseminados por el territorio. 3. ${ }^{\circ}$ El gobernador civil, que les sirve de órgano de comunicación y de instrumento. A esto se reduce fundamentalmente todo el artificio bajo cuya pesadumbre gime rendida y postrada la nación".

Joaquin Costa, Oligarquia y caciquismo como la forma actual del gobierno en España. Madrid, 1901.

Los comentarios guiados, donde aparecen unas cuestiones o preguntas cortas a desarrollar y que quedan representados por un $25,69 \%$ del total de la categoría:

ARBOR Vol. 187749 mayo-junio [2011] 599-615 ISSN: 0210-1963 
8.2. La tormentosa transición al liberalismo. Comentario de texto.

"Sumergida en el más profundo dolor por la súbita pérdida de mi augusto esposo y soberano, sólo una obligación sagrada, a que deben ceder todos los sentimiento del corazón, pudiera hacerme interrumpir el silencio que exigen la sorpresa cruel y la intensidad de mi pesar: La expectación que excita siempre un nuevo reinado, crece más con la incertidumbre sobre la administración pública en la menor edad del monarca: para disipar esta incertidumbre y precaver la inquietud y extravío que produce en los ánimos, he creído de mi deber anticipar a conjeturas y adivinaciones infundadas la firme y franca manifestación de los principios que he de seguir constantemente en el gobierno de que estoy encargada por la última voluntad del rey, mi augusto esposo, durante la minoría de la reina mi muy cara hija doña Isabel...".

(Del Manifiesto dirigido a la nación por la Reina Gobernadora M. ${ }^{a}$ Cristina de Borbón, Madrid 4 de octubre 1833, cfr. Manuel Rodríguez Alonso (editor), Los manifiestos políticos en el siglo XIX (1808-1874). Barcelona. Ariel Practicum. 1998, p. 102).

Cuestiones a desarrollar:

1) Explique el problema dinástico que planteó el fallecimiento de Fernando VII sin sucesión masculina; 2) ¿Qué apoyos hubo de buscar M. ${ }^{a}$ Cristina para sostener el trono de su hija? ¿Quiénes ayudaban a su rival?; 3) Resuma muy brevemente la guerra civil de los siete años o primera guerra carlista, y su desenlace.

Las preguntas concretas están formadas por enunciados con un cierto grado de complejidad en su redacción, pudiendo aparecer en ellas signos de interrogación. Además, el contenido por el que se pregunta queda limitado, evitando confusiones en cuanto al modo de abordarlo y en donde el discente no tiene opción a extenderse demasiado. Su representación en las preguntas estudiadas está en un 10,88\%. En ellas podemos encontrar dos subcategorías internas:

Las preguntas concretas de carácter general que incluyen un verbo que orienta el desarrollo de la respuesta, independientemente del formato (con o sin interrogación) y están representadas con un $89,16 \%$ del total de la categoria:
35.5. ¿Qué hizo Isabel II?

21.4. ¿Qué eran las tierras Manos Muertas? ¿Quiénes eran los propietarios de dichas tierras?

Y las de comparación, en las que en su redacción o enunciado se deja claro la actividad de comparar temas, sucesos, etc., y quedan representadas con un 10,84\% de la categoria:

74.2. Comparar las constituciones de 1812 y 1837.

118.4. Diferencias entre el partido moderado y el progresista.

Las cuestiones de vocabulario recogen preguntas en las que se les pide a los discentes que definan, de forma breve, conceptos o términos relacionados con la Historia. Normalmente, este tipo de preguntas constan de un número considerable de términos a definir y representan un 4,19\% del total de las cuestiones estudiadas:

59.4. Vocabulario: motín de Aranjuez, intendente, manifiesto de los persas, junta central suprema.

48.41. Vocabulario: absolutismo, diputado, carta otorgada, sufragio universal.

Para finalizar el estudio de la tipología de enunciados hemos de mencionar los mapas y las diapositivas. Los primeros son aquellas preguntas del examen que hacen referencia explícita al hecho de situar o localizar en un mapa eventos o lugares y en el total de las 763 preguntas estudiadas únicamente encontramos referencia a uno de ellos:

46.10. Traza una línea del tiempo y marca en ellas los acontecimientos más relevantes que ocurrieron entre 1868 y 1894

Las segundas son aquellas cuestiones que incluyen el comentario de fotografías, gráficos $u$ otros documentos, con la finalidad de relacionarlos con los contenidos trabajados en los procesos de enseñanza-aprendizaje. Al igual que los mapas, su presencia se constata en una única cuestión:

15.24. Diapositivas: atentado de Carrero Blanco y grabado de Fernando VII en la guerra de los Cien Mil Hijos de San Luis. 


\section{LAS PREgUNTAS dE EVALUACIÓN Y SU TEMÁtICA DE CONTENIDOS}

Realizado el estudio de la tipología de enunciados de las preguntas, y determinado que las preferidas por los docentes de la CARM de Historia de España para preguntar por el siglo XIX son las abiertas no guiadas, es el momento de presentar algunos de los resultados alcanzados en lo referido a los contenidos del mencionado siglo por los que el profesorado pregunta a su alumnado. Esta profundización en la temática de las cuestiones nos permite conocer qué acontecimientos ocurridos en la Historia del XIX son los que más importancia tienen a la hora de examinar al alumnado. Pero antes de pasar a un análisis en profundidad de éstas, es preciso matizar algunas cuestiones. Nuestro estudio se centra en 763 preguntas de exámenes; de ellas, 41 corresponden a cuestiones de vocabulario pero por ser estas preguntas de una naturaleza distinta al resto (incluyen en un mismo enunciado distintos conceptos con temáticas diversas), precisan un estudio diferenciado. Así pues, en primer lugar hacemos un análisis de los 722 enunciados $y$, posteriormente, pasamos a comentar brevemente las de vocabulario.
Para nuestro estudio conceptual hemos clasificado las preguntas atendiendo a todos y cada uno de los contenidos en ellas mencionados, resultando 4 categorías de estudio principales: política, economía, procesos sociales y cultura; dentro de las cuales hemos distinguido diferentes temáticas y sub-temáticas que clarifican las categorias amplias.

Los datos recogidos han puesto de manifiesto que son las cuestiones de indole política las que más se preguntan al alumnado, siendo representadas por un $78,39 \%$ del total. Le siguen, aunque con una importante diferencia, aquellas cuestiones que recogen los aspectos económicos, representados en un $17,73 \%$, y con un porcentaje mínimo encontramos las cuestiones de procesos sociales $(3,60 \%)$ y culturales $(0,28 \%)$. Estos datos se detallan a continuación con un análisis por temáticas.

\subsection{Política}

Los innumerables sucesos políticos transcurridos durante el siglo XIX constituyen el eje vertebrador de la enseñanza en la materia de Historia en el Bachillerato. Su presencia casi única $(78,39 \%)$ queda recogida en una amplia varie-

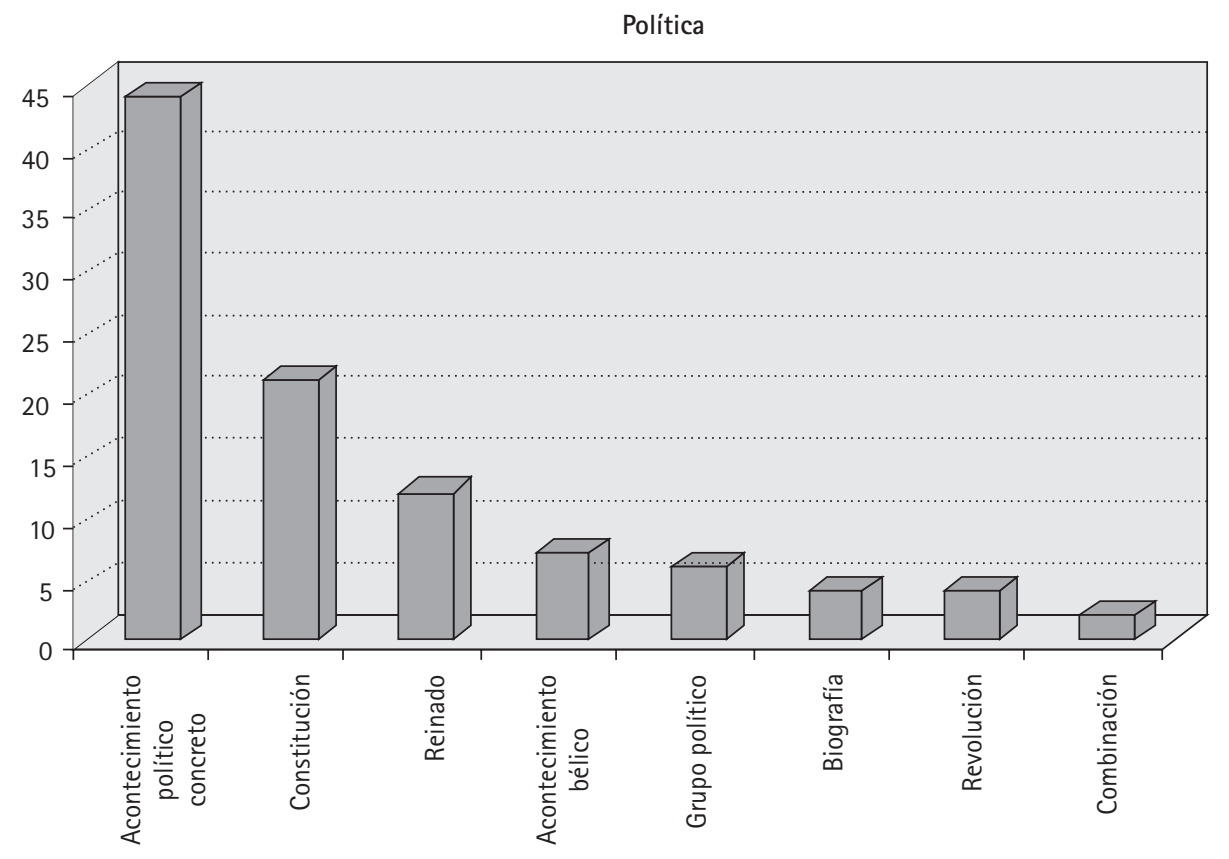

Figura 1. Porcentaje de representación de la temática política en las preguntas de exámenes. 
dad de temas que aglutinan desde los acontecimientos políticos concretos (categoría más representada), pasando por las constituciones, los diversos reinados y los sucesos bélicos y finalizando, con una presencia menor, con los grupos políticos, las biografías de los personajes más relevantes y las situaciones revolucionarias.

De entre estas temáticas organizadoras de los sucesos políticos, los acontecimientos políticos concretos y las constituciones merecen, por su número de preguntas, una breve referencia. Los primeros constituyen la temática más representada dentro de Política, con un 44\% del total (251 preguntas), e incluidas en ellos, encontramos cuestiones que hacen referencia a un suceso concreto ocurrido durante el período estudiado. Esta singularidad hace que esta categoría adquiera valor en sí misma, dada la variedad de temas que en las preguntas se trabajan. Así, de todos los hechos recogidos, el período del reinado de Fernando VII y la etapa de la Restauración son los más representados, con un $21 \%$ del total con cuestiones como las siguientes:

\subsection{Características de la Década absolutista.}

\subsection{El trienio constitucional.}

2.5. El reinado de Fernando VII: España entre la revolución y la reacción. Desarrollar: 1) ¿Qué se solicitó al rey Fernando VII a través de la publicación del Manifiesto de los Persas el 1de abril de 1814?; 2) ¿Cuáles fueron las consecuencias más importantes del pronunciamiento de Rafael de Riego en Cabezas de San Juan?; 3) ¿Qué significó la entrada en España de "Los Cien Mil hijos de San Luis"?; 4) Enumere las principales características de la década ominosa; 5) Consecuencias de la publicación de la "Pragmática Sanción".

9.1. El sistema político canovista.

46.13. ¿Qué motivos justificaron el "turno pacífico"? ¿Qué mecanismos se utilizaron para asegurar la alternancia política?

65.3. La restauración de la monarquía. Los problemas del régimen. El problema colonial y la crisis del surgimiento de los nacionalismos.
A estas dos temáticas, y para completar la descripción de algunos de los acontecimientos políticos concretos más referenciados, le siguen los sucesos transcurridos durante el reinado de Isabel II con un 17\% del total, así como las Cortes de Cádiz, con el 14\% del total; ambas abarcando una amplitud temática que recoge desde sucesos puntuales hasta importantes procesos políticos como son la implantación del liberalismo y el desarrollo políticoconstitucional de las Cortes de Cádiz:

2.1. La transición al liberalismo en España: el carlismo y la doble opción liberal y progresista.

18.10. La Formación del Estado Liberal.

55.5. El reinado de Isabel II: década moderada.

5.1. Las cortes de Cádiz: su obra reformadora.

37.1. Reformas de las Cortes de Cádiz.

53.2. Las cortes de Cádiz y su obra legislativa. Sectores sociales y políticos representados en las cortes.

La segunda categoría más recogida dentro de Política es la denominada "Constitución". Las preguntas encontradas en ella hacen referencia a aspectos contenidos en las diversas constituciones que durante el siglo XIX se promulgaron, y es que este siglo, caracterizado por la agitación política, fue de una importancia notable en este sentido. Todas ellas, tomaron como referencia lo legislado en las Cortes de Cádiz y la Constitución de 1812, siendo ésta considerada la primera de nuestras cartas magnas y la que marcó un camino al liberalismo tras los diversos reinados absolutistas en los que había estado sumida España. Su importancia en nuestra historia queda recogida por el número de las preguntas de los exámenes. Así, de un total de 124 cuestiones de constituciones, un 56\% (70 preguntas) hacen referencia a ésta. Su redacción puede variar como muestran los siguientes ejemplos:

81.7. Constitución de 1812: características, relación con otras constituciones y valoración.

91.6. Principios de la Constitución de 1812.

115.1. Constitución de 1812 . 


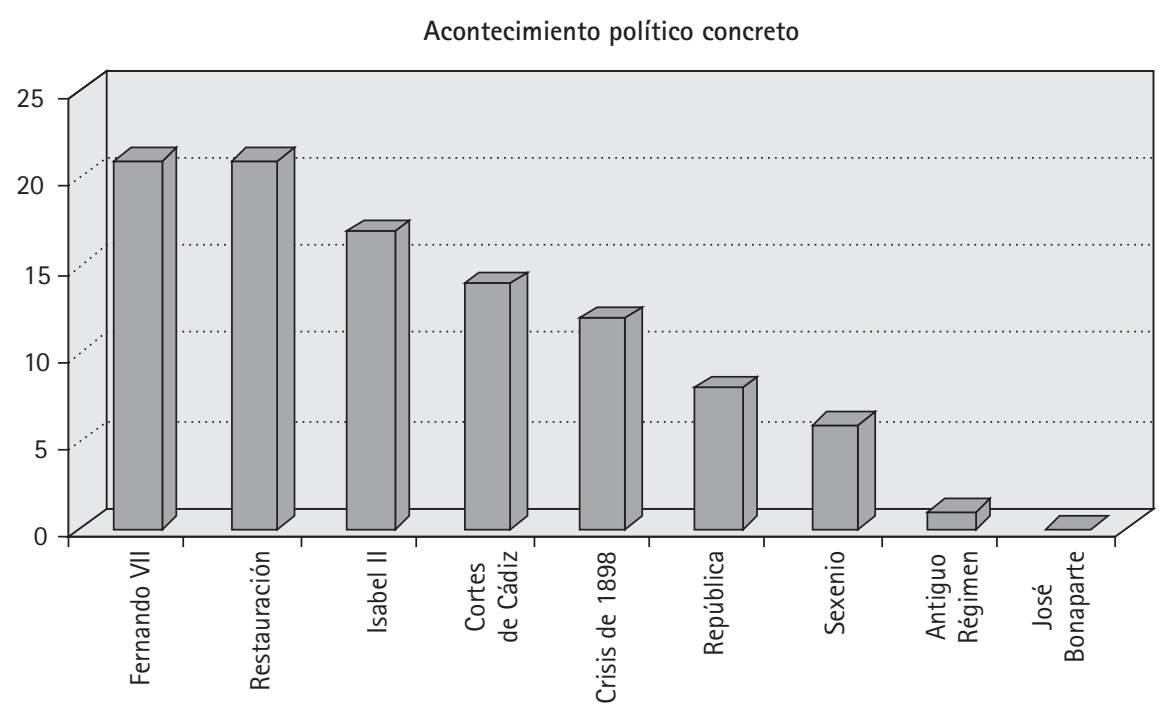

Figura 2. Porcentaje de representación de la temática acontecimiento político concreto en las preguntas de exámenes.

El resto de subcategorias que forman el apartado de Politica: reinado, acontecimiento bélico, grupos políticos, biografías, revolución y la combinación de ellas; presentan un porcentaje de representación bastante menor pero no por ello menos importante.

\subsection{Economía}

La economía del siglo XIX representa la segunda temática en importancia reflejada en las preguntas de los exámenes. Formada por 128 cuestiones $(17,73 \%$ del total) trata diversas temáticas, desde la agricultura hasta los transportes, pasando por los cambios demográficos, los procesos desamortizadores, la hacienda, las políticas industriales y la política fiscal.

Durante el siglo XIX se produjeron en España una serie de transformaciones que cambiaron el sistema económico. Éstas hacen que se inicie el camino abierto por los países industrializados y los principales cambios afectarán a las medidas jurídicas que tiene como objetivo eliminar la estructura que define el Antiguo Régimen. Estos cambios en la titularidad de la tierra se modifican, en gran parte, gracias a las desamortizaciones y esto queda reflejado en el gran número de preguntas que encontramos en los exámenes haciendo referencia a esta temática (45\%). Pero no sólo las desamortizaciones serán importantes en este período. La industria (29\%) y su desarrollo también aparecen con frecuencia. Ambas categorías forman el grueso de las preguntas ( $84 \%$ del total de la categoria), tal y como recoge la siguiente figura 3 .

Especificando lo comentado con anterioridad, la renovación de la titularidad de la tierra sufre modificaciones gracias a los procesos desamortizadores del XIX. Éstos se inician en 1798 con la desamortización de Godoy, una disposición legal que afecta principalmente a la Iglesia. La segunda desamortización, y la más famosa históricamente, es la de Mendizábal, puesta en marcha en 1836. Esta es la desamortización eclesiástica general que, por la institución a la que afecta, ha sido muy polémica y fue posible gracias al acceso al poder de los liberales. Pero la desamortización que más trascendencia tuvo para la puesta en el mercado de tierras nuevas, y para la adquisición por parte de la burguesía de un patrimonio terrateniente, fue la desamortización de Madoz en 1855. Ésta fue la desamortización civil y general que puso en el mercado numerosas tierras que hasta el momento habían sido de uso comunal.

Las preguntas de desamortización en los exámenes forman la categoría con mayor representación, un 46,03\%, a través de sus 58 cuestiones. Podemos señalar que estas 


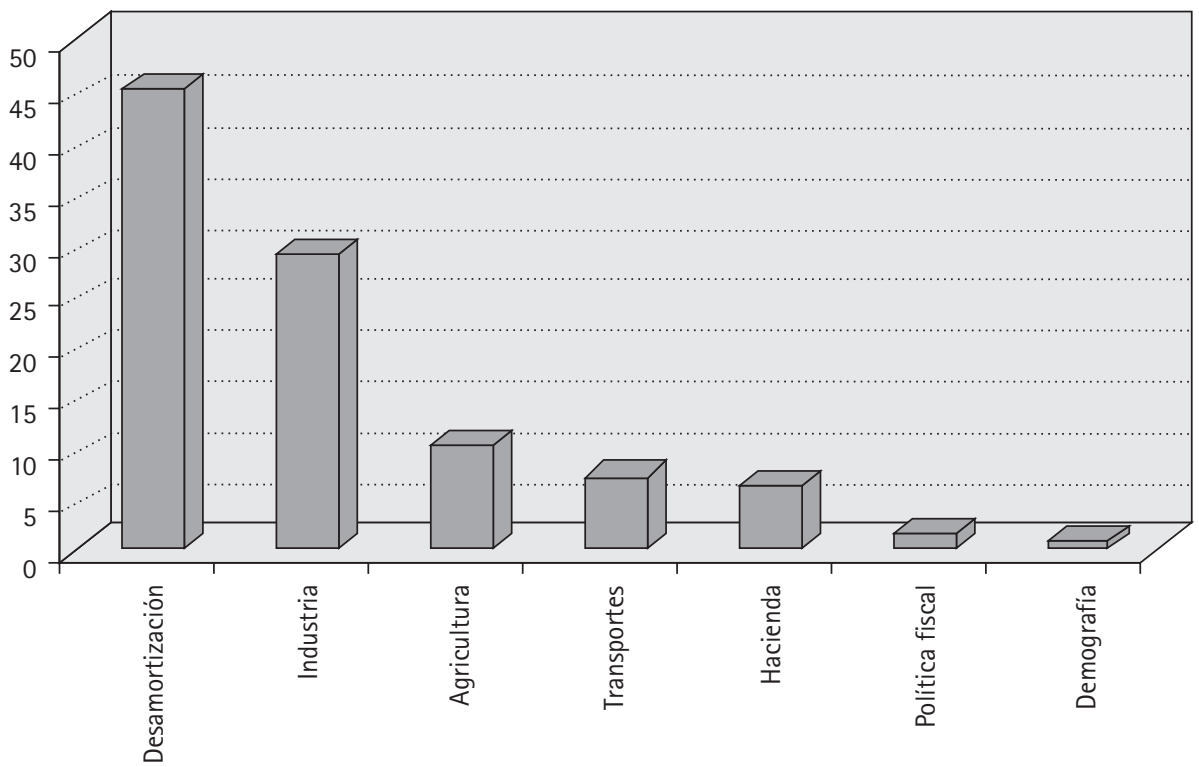

Figura 3. Porcentaje de representación de la temática economía en las preguntas de exámenes.

preguntas son utilizadas por el profesorado como la mejor forma de trabajar los aspectos económicos del siglo XIX debido a su singularidad y especificidad. En ellas podemos distinguir aquéllas que tratan la desamortización de Madoz o Mendizábal de forma individual o si por el contrario se pide una explicación de la ley de las desamortizaciones, con un tratamiento más amplio y general.

68.5. Las transformaciones agrarias: la doble desamortización eclesiástica y civil.

11.6. La desamortización de Mendizábal y Madoz.

29.16. La doble desamortización eclesiástica y civil: Mendizábal y Madoz.

En la segunda categoria en importancia dentro de Economía podemos ubicar todas aquellas cuestiones que hacen referencia a procesos industriales acontecidos en el siglo XIX. Concentran un 29,37\% (37) de las cuestiones económicas y pueden concretarse su mayoría en torno a la Revolución Industrial, con menor número de referencias a infraestructuras o al crecimiento textil experimentado en esta centuria. En ocasiones la temática de la revolución industrial va acompañada de las medidas económicas provocadas por la misma, relacionando así en una misma pregunta las dos temáticas, dando la opción al alumnado de tratarlo de forma global haciendo uso de su visión de conjunto.

35.3. Etapas de la revolución industrial.

48.17. Incorporación tardía de España a la revolución industrial.

60.15. El debate proteccionismo librecambismo.

Al igual que ocurre con la temática de Política, el resto de categorias relacionadas con la Economía presentan un porcentaje de representación menor.

\subsection{Procesos sociales y cultura}

Los procesos sociales y culturales vividos durante el XIX son, sin duda, los grandes olvidados. Su presencia en el cómputo general de las preguntas estudiadas apenas llega 
al 5\%, no recogiendo por tanto, las importantes consecuencias que tanto los procesos políticos como económicos tienen para el desarrollo social y cultural de la población española del XIX.

De este modo, la temática de procesos sociales queda escuetamente representada en las preguntas con un 3,60\% (26 cuestiones) del total. Si bien es cierto que su presencia es escasa, los temas que abordan son variados, recogiendo desde los primeros movimientos sociales hasta la sociedad española durante el período de la Restauración, pasando por el surgimiento de los movimientos obreros, cantonales y nacionalistas.

4.13. La sociedad española durante la Restauración.

7.16. El movimiento obrero.

22.7. Los primeros movimientos sociales.

22.11. El surgimiento de los nacionalismos.

En cuanto a la categoría de cultura, únicamente hemos encontrados 2 cuestiones:

25.5. Cultura de la restauración: conservadurismo y regeneracionismo.

45.4. ¿Qué es lo que Machado quiso expresar en el siguiente poema (La Saeta) dentro del pensamiento de la generación del 98 ?

Esta ausencia de preguntas relacionadas con los aspectos culturales y sociales de la España del siglo XIX, es un dato revelador de la importancia que otros temas tienen en el estudio de este siglo. No podemos olvidar que todo acontecimiento histórico, ya sea político o económico tiene repercusiones en los sucesos culturales y sociales que se viven con posterioridad. Así, y como bien señala el caso 45.4., la guerra de Cuba de 1898 tuvo importantes consecuencias en los acontecimientos culturales de la época. De ella nace la Generación del 98, nombre con el que se agrupa a los escritores, ensayistas y poetas españoles que se vieron afectados por la crisis moral, política y social que vivía España tras la pérdida de las colonias de Ultramar. Su relación con los acontecimientos políticos es imprescindible para comprender profundamente los cambios políticos que en este período se vivieron. Por último, es preciso reconocer que el concepto de cultura es fundamental para las disciplinas que se encargan del estudio de la sociedad y la Historia es una de ellas.

Realizado un análisis minucioso de cada categoría, es preciso realizar una diferenciación entre las preguntas recogidas del año 2002/2003, legisladas desde el Decreto del Ministerio de 1992, y las recogidas en el año 2003/2004 y propuestas desde el Decreto de la CARM de 2002.

Aunque el orden de importancia de las temáticas es el mismo (política, economía y procesos sociales y cultura), sus porcentajes varían. La categoría de política estaba representada en las preguntas de 2002/2003 con un 77,50\%, mientras que en el año 2003/2004 se produce un ligero aumento de su presencia llegando hasta el $82,79 \%$. Sin embargo, en la temática económica el cambio de decreto conllevó un descenso del porcentaje de estas preguntas, bajando de un 18,17\% hasta un 15,57\%. Esta misma tendencia de bajada es la sufrida por los procesos sociales y la cultura, ésta última llega incluso a desaparecer en las preguntas de 2003/2004.

\subsection{Vocabulario}

Como hemos comentado anteriormente, las preguntas de vocabulario tienen una naturaleza distinta al resto de las cuestiones. En ellas, el profesorado recoge bajo un enunciado genérico varios términos que el alumnado debe definir. Por ello, su análisis debe ser diferenciado ya que en una misma cuestión podemos encontrar temáticas diversas. Así, de 763 cuestiones que forman nuestra base de datos, 41 las calificamos como de vocabulario, representando el 5,37\% del total de las preguntas estudiadas, en base a las cuales, hemos realizado una base de datos distinta, separando cada concepto y atendiendo a su temática. Así, considerando cada término como una pregunta distinta encontramos 172 . El análisis realizado a cada una de ellas, ha puesto de manifiesto que el $84 \%$ de las cuestiones recogen aspectos políticos, manteniendo la tónica del resto de las preguntas. A estos acontecimientos políticos, le sigue con una importante diferencia la economía (12\%), los procesos sociales (3\%) y una cuestión del siglo XVIII, desapareciendo en ellas los procesos culturales del XIX.

Haciendo una escueta especificación de estos resultados generales, es preciso señalar que al igual que en el grueso

ARBOR Vol. 187749 mayo-junio [2011] 599-615 ISSN: 0210-1963 
de preguntas, los términos vinculados a acontecimientos políticos concretos serán los más demandados por el profesorado en sus preguntas de examen, seguidos de cuestiones en torno a grupos políticos y biografías, tal y como muestran los siguientes ejemplos:

6.11. Relaciona cada término con su fecha correspondiente y ordénalos, según su trascendencia a lo largo de la historia: congreso de Verona.

6.11. Relaciona cada término con su fecha correspondiente y ordénalos, según su trascendencia a lo largo de la historia: Pragmática Sanción.

56.8. Explica brevemente: Pi y Margall.

142.4. Vocabulario: isabelinos.

142.4. Vocabulario: carlistas.

110.3. Vocabulario: moderados.

Los términos económicos, segundos en representación con un $12 \%$ del total de las preguntas analizadas, recogen cuatro temáticas. De ellas, las que hacen referencia a la hacienda representan un 52\% del total de la categoría, seguidas por las que recogen cuestiones sobre desamortización con un $38 \%$, y finalizando, con un $5 \%$ cada una, las temáticas de política fiscal y transportes:

55.14. Explica brevemente: Banco de España.

55.14. Explica brevemente: arancel Figuerola.

56.4. Explica brevemente: Madoz.

6.11. Relaciona cada término con su fecha correspondiente y ordénalos, según su trascendencia a lo largo de la historia: ley de ferrocarriles.

Y para finalizar con el análisis de las preguntas, recogemos las cuestiones de vocabulario referidas a procesos sociales, representadas en un 3\% del total de las estudiadas. Al igual que en el resto de las cuestiones, su presencia es escasa:

14.6. Conceptos: sociedad de clases.
18.1. Escribe los significados de los siguientes términos: pecheros.

55.25. Explica brevemente: Antiguo Régimen.

\section{Conclusiones: la Repercusión de la eVAluación Y SU CONTENIDO EN EL APRENDIZAJE DE LOS DISCENTES}

La concepción del Bachillerato dentro de la LOGSE quedó definida como la etapa que permite al alumnado la adquisición y dominio de unos conocimientos capaces de hacerle tomar decisiones propias referidas a su educación. En este ciclo post-obligatorio, la transmisión de valores y el enriquecimiento académico y personal se configuran como ejes predominantes de la enseñanza. En él, aparece una materia obligatoria capaz de aportar al discente el conocimiento del pasado y la repercusión de éste en la actualidad: la Historia. Concebida como una disciplina de carácter eminentemente social, su ámbito de estudio estará determinado por las disposiciones legales realizadas al respecto. Así, en aplicación de los principios contenidos en la LOGSE, el R. D. de 1992 postuló el estudio de los discentes a una etapa específica de ésta, la España contemporánea. El desarrollo conceptual de los siglos XIX y $X X$ debía poner al alcance del alumnado la comprensión de distintos fenómenos políticos, económicos, sociales y culturales que aportasen globalidad a su formación y que le sirviesen de base en la toma de decisiones futuras. La ampliación de contenidos introducida en el año 2000, y especificada para la CARM en el Decreto de 2002, pone de manifiesto la necesidad de presentarle al estudiante de Bachillerato todos y cada uno de los períodos vividos por la población española desde el proceso de hominización hasta el estado del bienestar actual.

El análisis realizado de ambos decretos a nivel conceptual pone de relieve importantes cambios. El primero de ellos, como ya hemos comentado, es el relativo a la amplitud de las épocas de estudio. La segunda diferencia reseñable podemos concretarla en la mención que realiza el R. D. de 1992, tanto a nivel de objetivo, como conceptual y evaluativo, de las "Fuentes y procedimientos para el conocimiento histórico. Análisis y utilización crítica de fuentes y material historiográfico diverso. Contraste de interpretaciones his- 
toriográficas y elaboración de síntesis integrando información de distinto tipo." (R. D. 1178/1992, anexo 1, Historia). Que se utilizan para comprender el conocimiento histórico, contenido que es suprimido en el Decreto de 2002 de la CARM. En ninguno de los enunciados de las preguntas se hace referencia a las fuentes históricas, su utilización y el tipo de información que pueden aportar. Y ello, a pesar de que en esta materia su uso es fundamental para el acercamiento crítico a la realidad estudiada y su ausencia en el aula desemboca en una explicación histórica de los docentes sin recurrir a los elementos que han permitido construirla. Esta carencia impide que el alumnado genere conocimiento propio a partir de ellas.

Pese al importante cambio introducido en los contenidos, los criterios evaluativos se han mantenido intactos. En este sentido, es preciso mencionar que los objetivos evaluativos expuestos desde el R. D. de 1992 tenían un carácter amplio y globalizador que ha sido mantenido en disposiciones legislativas posteriores. Junto a esta continuidad evaluativa remarcamos la permanencia de la corriente historiográfica en la que se sustentan los contenidos propuestos objeto de estudio. En ambos casos, y del estudio del siglo XIX, se percibe una fuerte influencia de la escuela de Annales, y algunas de las corrientes surgidas de ella, pero su orientación es parcial, pues aunque el estudio que se lleva a cabo es de períodos amplios, priman los acontecimientos políticos por encima de los económicos, culturales o sociales, y hay escasas preguntas en las que se inquiere por la relación entre ellas.

Tomando como base de nuestro trabajo estas ideas, el objeto de estudio ha estado vinculado a la evaluación del siglo XIX en la materia de Historia de España de 2. Bachillerato y nos ha permitido profundizar en lo que está ocurriendo en las aulas en esta materia y en este nivel educativo, pues en cierta medida, al acceder a los exámenes que lleva a cabo el profesorado, estamos adentrándonos en aquello que el docente considera importante tanto para la enseñanza de la Historia como para el devenir en el aprendizaje de su alumnado.

Por otra parte, el acercamiento a esta práctica evaluativa ha puesto de manifiesto una realidad cargada de incertidumbres, cambios y discontinuidades, consecuencia de la diversidad de funciones y expectativas que se espera que ésta cumpla por parte de los dos agentes educativos principales: docentes y alumnado.
Como se ha podido constatar, dentro de los procesos evaluativos llevados a cabo en las aulas de Historia de España de la CARM, la técnica por excelencia utilizada por el profesorado para obtener información del proceso de enseñanza-aprendizaje, son los exámenes, y de forma mayoritaria, los de redacción son los más utilizados. Estos exámenes nos han permitido conocer qué tipología de enunciados y qué contenidos los conforman y nos han dado la base para contrastar la realidad de las aulas con lo legislado desde las instancias educativas.

Como es ampliamente conocido, el docente se apoya en unos referentes en base a los cuales concreta todo su quehacer evaluativo. Éstos quedan recogidos en los decretos de currículo, las pruebas de acceso a la Universidad y su propia experiencia unida a las directrices dadas por los departamentos. Más concretamente, son los contenidos y los criterios de evaluación establecidos en los curricula y las preguntas de las pruebas de selectividad los que marcan los enunciados de las preguntas de examen.

Ante esta circunstancia, es preciso ejemplificar un examen tipo de la materia de Historia en $2 .^{\circ}$ de Bachillerato. Compuesto generalmente, y tal y como recoge Martínez Molina (2008), por 4 preguntas en cada uno, los enunciados de éstas serán predominantemente de tipología abierta, no guiada, donde al discente se le da un tema y debe desarrollarlo sin un guión marcado por el docente. En estas preguntas tienen una importancia vital todas aquéllas que hacen referencia a sucesos políticos, dejando en un porcentaje de representación menor lo que concierne a temas económicos, sociales o culturales. Esta ejemplificación pone de manifiesto que pese al importante cambio legislativo experimentado en los cursos objeto de estudio, 2002-2003 y 2003-2004, los hechos transcurridos durante este siglo son preguntados de forma similar.

Nuevamente señalamos que son los contenidos que hacen referencia a los sucesos políticos aquéllos que predominan en los exámenes (78,39\%), y de forma específica, las cuestiones relacionadas con la etapa del reinado de Fernando VII son las más solicitadas por el profesorado. El hecho de que se mantenga esta temática pese al cambio producido deja entrever que si bien en ambos decretos prima la corriente historiográfica de Annales, al igual que en las preguntas del profesorado, la presencia de la política es

ARBOR Vol. 187749 mayo-junio [2011] 599-615 ISSN: 0210-1963 
demasiado elevada para dar paso a otras cuestiones de carácter social, económico o cultural.

Reseñamos, por tanto, que trabajar la historia de un pais sin relacionar hechos políticos con su trascendencia en el plano de la vida social lleva a estudiarla de forma sesga$d a$, cuando su razón de ser sería hacerlo tomando como referencia otras disciplinas afines, tales como Sociología, Antropología, Economía, etc., que pueden aportar un modo holístico y comprensivo de concebir la Historia de este siglo.

Para finalizar con nuestro trabajo, consideramos también pertinente reseñar la relevancia conceptual de lo enseñado en el siglo XIX. Si este término lo asociamos con el contenido que es preguntado a los discentes en sus exámenes, podriamos, a priori, determinar que será más importante cuantas más veces se pregunte. Siguiendo con este planteamiento, constatamos que la Constitución de 1812, Fernando VII y el período de la Restauración son los más destacados del siglo XIX a la vista de las frecuencias con lo que son preguntados, mientras que los sucesos menos significativos del siglo son aquéllos relacionados con acontecimientos culturales, política fiscal y demografía, entre otros muchos.
Este resultado nos devuelve a la idea de que para nuestros docentes, y en base a lo establecido en los decretos y las PAU (éstos servirán de guía a los otros), estos tres temas tienen preeminencia desde el ámbito de la Historia, pues son los núcleos articuladores del acontecer de toda esta centuria, marcada por importantes cambios, donde se abole el Antiguo Régimen, se superan importantes guerras, se configura un sistema de gobierno liberal, se instaura una república y se acaba con una restauración, por señalar sólo algunos. Pero esta relevancia conceptual propuesta en el aula olvida una de las características más importantes de la disciplina histórica: su desarrollo sustentado en el compendio de diversas y múltiples disciplinas.

Podemos finalmente mantener que del siglo XIX una constitución (1812), un personaje (Fernando VII) y un proceso (la Restauración), constituyen el bagaje por el que se les examina (teóricamente se les enseña y ellos estudian), a los discentes al terminar su formación anterior a la universidad o a la formación profesional. Sin embargo, sabemos poco de la trascendencia y significado que dichos contenidos alcanzan en los discentes. Sin duda profundizar en ese campo es objeto de otros estudios, otras técnicas y otros modos de indagarlos, apasionantes por el significado de la Historia, la realidad del alumnado y la formación de los docentes.

\section{NOTAS}

1 Lista de siglas utilizadas en este texto: CARM: Comunidad Autónoma de la Región de Murcia; LOGSE: Ley Orgánica de Ordenación General del Sistema Educativo; PAU: Pruebas de Acceso a la Universidad; R. D.: Real Decreto.

El presente trabajo ha sido realizado en el seno del proyecto "Diseño y desarrollo de los proyectos Curriculares de Historia en Bachillerato de la Comunidad Autónoma de la Región de Murcia" (03003/PHCS/05). Financiado por la Fundación Séneca de la CARM.

\section{BIBLIOGRAFÍA}

Alonso Tapia, J. (1997): Evaluación del conocimiento y su adquisición. Volumen I, Ciencias Sociales, Madrid, CIDE.

Beltrán Tena y Rodríguez Diéguez, J. L. (2004): "Clasificación de exámenes escritos". Voz en Salvador Mata, F., Rodríguez Diéguez, J.L. y Bolivar Botía, A. Diccionario Enciclopédico de Didáctica (Vol. 1), Archidona (Málaga), Aljibe.

Bolívar A. y Rodríguez Diéguez, J. L. (2002): Reformas y retórica. La reforma educativa de la LOGSE, Málaga, Aljibe.

Gimeno Sacristán, J. y Pérez Gómez, A. (1992): Comprender y transformar la enseñanza, Madrid, Morata.
Recibido: 10 de junio de 2010

Aceptado: 3 de diciembre de 2011 
Gullickson, A. R. (1982): "The Practice of Testing in Elementary and Secondary Schools", Unpublished report, ED229391.

Hargreaves, A. y Ryan, J. (1998): Una educación para el cambio. Reinventar la educación de los adolescentes, BarceIona, Octaedro.

Herman, J. y Dorr-Bremme, D. W. (1984): Testing Assessment in American Public Schools: Current Practices and Directions for Improvement, Los Ángeles, Center for the Study of Evaluation, University of California at Los Angeles.

Lafourcade, P. D. (1972): Evaluación de los aprendizajes, Madrid, Cincel.

Martínez Molina, M. ${ }^{a}$ E. (2008): La evaluación de Historia de España en COU y Bachillerato en la Comunidad Autónoma de la Región de Murcia (1993-
2004), Tesis Doctoral no publicada, Facultad de Educación, Universidad de Murcia.

Martínez Valcárcel, N. (2003): Didáctica General. Pedagogía, Murcia, Serie Tresmiles.

Martínez Valcárcel, N.; Miralles Sánchez, P. y Navarro Medina, E. (2009): "Análisis de cómo los docentes plantean el inicio de las clases desde la perspectiva del alumnado", Revista de Educación, n. ${ }^{\circ}$ 348, pp. 413-433.

Ortega Carrillo, J. A. y Sánchez Domínguez, J. (1996): "El bachillerato LOGSE visto desde una perspectiva profesional: dificultades e incertidumbres en su implantación", I Congreso Nacional de Bachillerato, Granada.

Piaget, J. (1985): A dónde va la educación, Barcelona, Editorial Teide.
Rodríguez Diéguez, J. L. (1994): "Los componentes del currículo", Didáctica general: un enfoque curricular, Alcoy, Editorial Marfil.

Rodríguez Neira, T. (Coord.) (2001): "Pruebas libres", La evaluación en el aula, Siero (Asturias), Ediciones Nobel.

Santos Guerra, M. A. (1993): La evaluación: un proceso de diálogo, comprensión y mejora, Archidona (Málaga), Aljibe.

Villa Arocena, J. L. y Alonso Tapia, J. (1996): “Cómo se evalúa el aprendizaje en enseñanzas medias?", Revista de Ciencias de la Educación, 168, 473-503.

Wragg, E.C. (2003): Evaluación y aprendizaje en la escuela secundaria, Barcelona, Paidós Educador.

Zabalza, M. A. (1991): Diseño y desarrollo curricular, Madrid, Narcea. 